\title{
Bone Induction by Demineralized Dentin Matrix in Nude Mouse Muscles
}

\author{
Kyung-Wook Kim \\ Department of Oral and Maxillofacial Surgery, Dankook University College of Dentistry
}

\begin{abstract}
Purpose: This study examined the osteoinductive activity of demineralized human dentin matrix for nude mice.

Methods: Twenty healthy nude mice weighing about 15 to $20 \mathrm{~g}$ were used for study. Demineralized human dentin matrix was prepared and implanted into the dorsal portion of nude mice (subcutaneous), which were sacrificed at two, four, and eight weeks after demineralized dentin matrix grafting and evaluated histologically by H\&E and Masson trichrome staining. The specimens were also evaluated histomorphometrically.

Results: The demineralized dentin matrix induced bone and cartilage formation independently in soft tissues. Histological examination showed bone-forming cells such as osteoblasts and fibroblasts at two, four, and eight weeks.

Conclusion: These results suggest that demineralized human dentin matrix has osteoinductive ability, and is a good alternative to autogenous bone graft materials.
\end{abstract}

Key words: Dentin, Demineralized human dentin, Bone induction

\section{Introduction}

In most edentulous patients who need dental implant, there is also no alveolar bone. Therefore, in many cases alveolar bone restoration is needed for implant treatment. Bone graft materials are usually divided into autogenous bone, allogenic bone, xenogenic bone and synthetic bone according to donor site. Autogenous bone is regarded as the 'gold standard' of bone graft because of its safety and effectiveness. However, additional surgery is needed at the donor site, presenting a risk of complications such as infection or pain, and the collection amount has a limit[1-3]. Also, with allogenic bone and xenogenic bone there is concern of contagion, and osteogenesis effect that is lower than autogenous bone[4-6]. There can be occult infections such as hepatitis or tuberculosis in the cadaver from which allogenic bone is obtained, while the bovine bone often used as the source of xenogenic bone can carry mad cow disease or other zoonoses. Synthetic bone has no risk of disease contagion as it is a manufactured product, but is capable only of osteoconduction[7]. Therefore, there is active research on new bone graft materials with bone regeneration ability equivalent to autogenous bone but without the limitations of allogenic, xenogenic and synthetic bone.

Urist proved demineralized dentin has the same osteo-

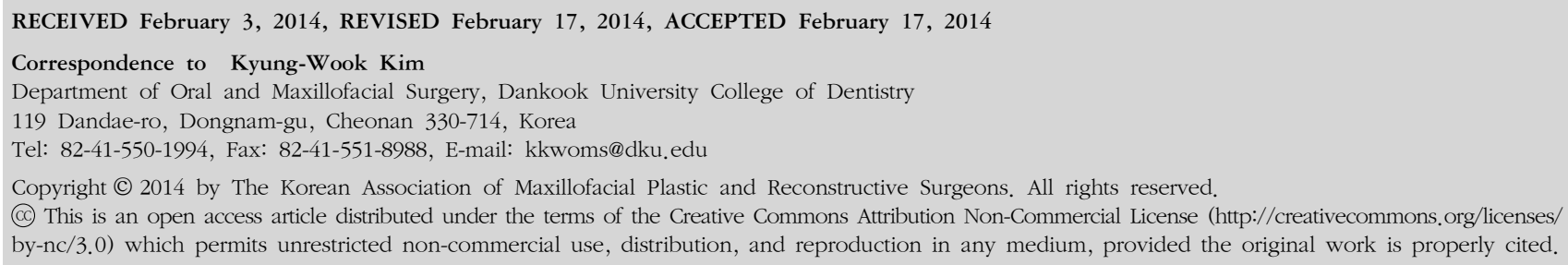


conduction effect as demineralized bone[8-10]. As this was a strong evidence for the existence of organic substance in demineralized dentin, the existence of tooth protein was discovered by the patent of a method to extract tooth protein from extracted teeth[11]. Since then, research on demineralized dentin has continued. Murata et al.[12] reported successful development of a device that generates bone from a tooth that was demineralized in the dental office. Kim et al.[13] announced they successfully processed tooth as bone graft material for clinical application.

Autogenous tooth bone graft material consists of $55 \%$ minerals and $45 \%$ organic matters. The minerals have excellent osteoconductivity as they are made of low crystalline apatite[14]. Organic matters have many bone growth factors including type I collagen and bone morphogenetic protein (BMP)[13]. Therefore, autogenous teeth bone graft material has bone healing effects only when adjacent to an autogenous bone graft. There is research that the tooth dentin, especially, induces bone formation because it has abundant type I collagen and well-developed dentinal tubules[13-17].

This study histologically observes the osteoinductive ability of demineralized dentin matrix (DDM) manufactured from the extracted tooth of human and implanted in the muscle of nude mice, and furthermore, to observe superiority of autogenous teeth bone graft material.

\section{Materials and Methods}

\section{Research materials}

\section{1) Laboratory animal}

Twenty four-week old nude mice (weight: about 15 20 g) from Orient Bio Inc. (Seongnam, Korea) were used for the animal model portion of this study. After a five day adjustment period, we checked that the mice were normal, then began the experiment. The animals were freely fed with hard food and water at $22^{\circ} \mathrm{C}$ temperature and 12 hours light contrast cycle. We observed the policy of Korean Association for Laboratory Animal Science and regulations related to animal experiment.

\section{2) Preparation of demineralized dentin matrix from human}

An extracted human tooth soaked in 70\% ethyl alcohol was sent to a specialized agency (Korea Tooth Bank Co., Seoul, Korea), and foreign substances such as soft tissue or tartar were removed. The tooth was divided into crown and root and crushed. One to two mm crushed particles were soaked in distilled water and hydrogen dioxide solution, and the remaining foreign substances were removed by ultrasonic cleaner. The cleaned particles were dehydrated with ethyl alcohol and went through defatting using ethyl ether solution. The particles were then lyophilized and disinfected with ethylene oxide gas, packed and sent back to the laboratory to be used in implanting.

\section{Research method}

\section{1) Intramuscular implantation}

We gave the animals general anesthesia through intraperitoneal administration of pentobarbital sodium (Nembutal, $43 \mathrm{mg} / \mathrm{kg}$; Dainabot Co., Tokyo, Japan) that had been diluted in sterile water for injection, then disinfected and isolated the surgical site. An incision was made in dorsum and a pouch was formed in muscle at both sides. We implanted DDM into a pouch of muscle, sutured the cut with nylon thread, and applied antibiotic ointment to prevent infection.

\section{2) Manufacturing of tissue specimen and observation}

Laboratory animals were sacrificed at two weeks, four weeks, and eight weeks after DDM grafting. Immediately after the sacrifice, DDM and its neighboring tissue was collected. The collected tissues were fixed in a 10\% buffered formalin for over 10 days, demineralized with formic acid, and dehydrated with ethanol. Finally, the tissues were embedded in paraffin and the specimens were completed. They were evaluated histopathologically after H\&E, Masson trichrome (MT) staining, and observed with Olympus BX-51 (Olympus Co., Tokyo, Japan).

\section{3) Histomorphometric analysis}

The osteoinductive ability of DDM implanted in soft tissue is evaluated by checking the graft material for the presence of a chemotactic factor like BMP can develop the undifferentiated mesenchymal cells into chondroblast and osteoblast. Therefore, to count the DDM's neighboring bone formation cells (osteoblast, fibroblast), we divided the number of dentin particle's neighboring bone forming 
cells by the number of dentin particle observed in the specimen. We charted the result on a graph (Fig. 1).

\section{Results}

\section{Histological observation}

Histopathological observation revealed bone forming cells surrounding the bone graft material, DDM particles, and small quantities of new bone tissue at two, four, and

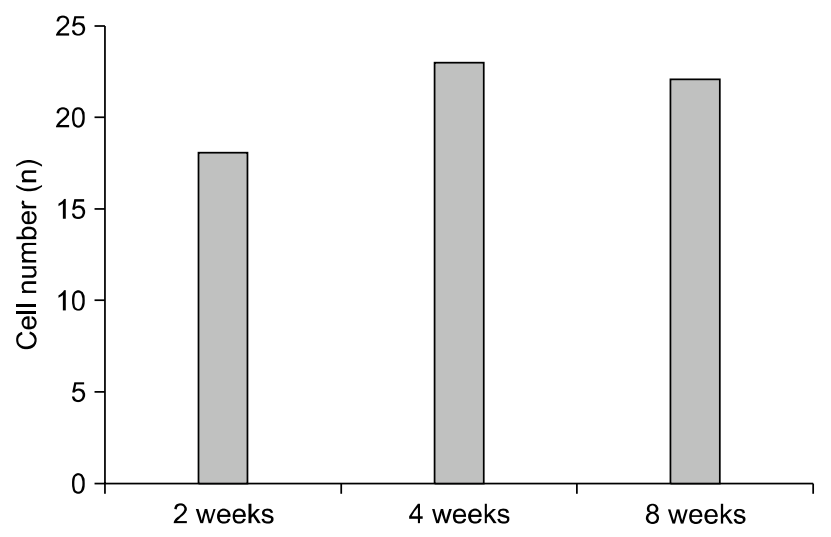

Fig. 1. Morphometry of bone-forming cells.

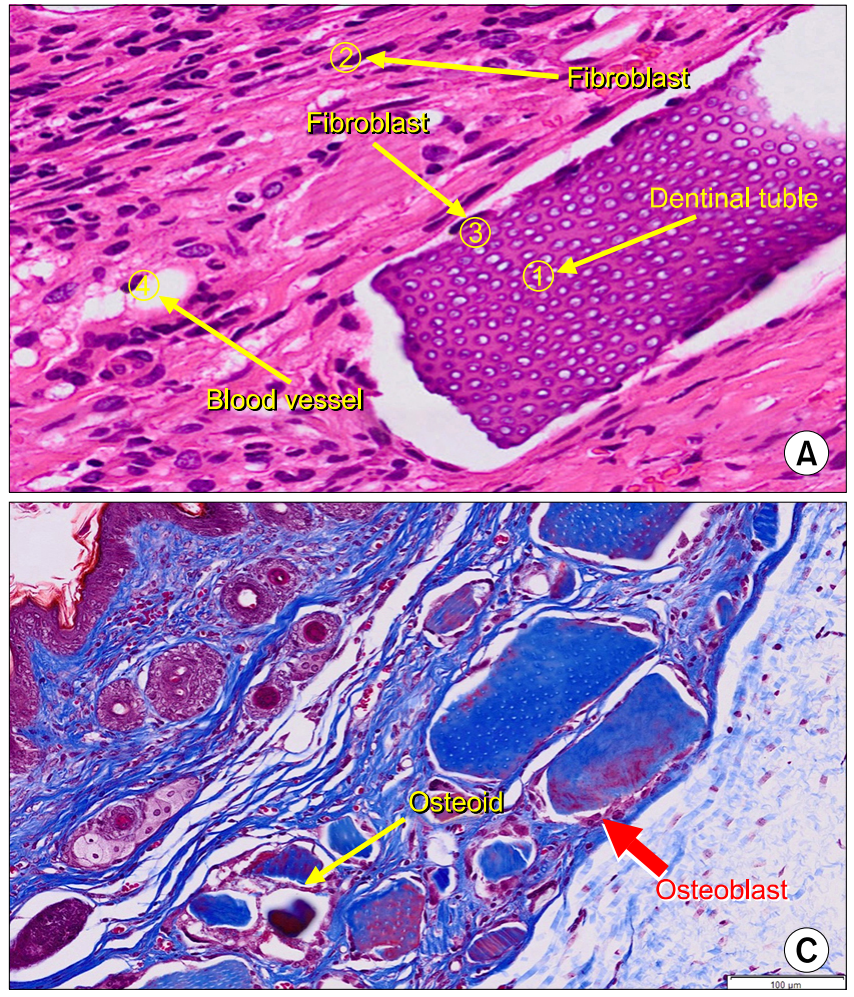

eight weeks. At two weeks, fibroblasts and blood vessels were observed in soft tissue adjacent to dentin particles, and fibroblasts were attached to dentin particles. We observed fibroblasts attached in thin fibrous membranes surrounding particles forming new bone, and osteoblast and osteoblast-like cells were present in H\&E stained photography (Fig. 2). At four weeks, fibrous tissues (reservoirs of angiogenesis as well as stem cell supplies), were well organized between the particles, and collagen matrix was observed, likely formed by neighboring osteoblast cells. Vascularized tissues were observed and osteocytes were surrounded by bone matrix newly formed around bone graft material (Fig. 3). At eight weeks, more activated osteoblastic activities developed, and newly formed bone tissue was gradually calcified. Osteoblastic cells gradually formed from soft tissue toward bone graft material and new bone tissue was directly formed around the particle of DDM, which was an osteoinduction (Fig. 4).

In eight week specimens, the cartilage connecting DDM particles like a bridge was observed (Fig. 5). The cartilage and bone formed independently and the formation of bone marrow was also observed.

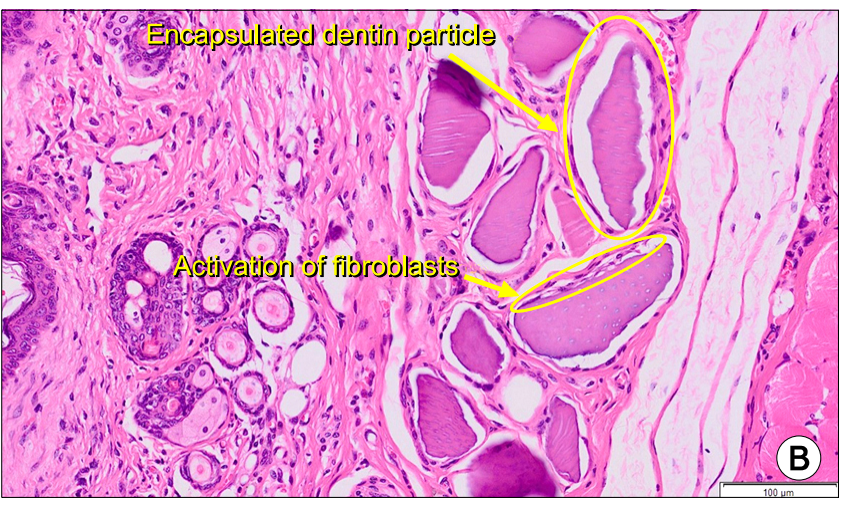

Fig. 2. (A) Histological finding of demineralized dentin matrix (DDM) after 2 weeks (H\&E, $\times 500)$ : Dense fibrous tissues with moderate blood vessels and newly attached fibroblasts are observed around dentin particles. (1) Dentinal tuble. (2) Fibroblast. (3) Fibroblast. (4) Blood vessel. (B) Histological finding of DDM after 2 weeks $(\mathrm{H} \& \mathrm{E}, \times 200)$ : Well-encapsulated dentin particles within cell supplies and cellular activities on the surface of dentin, which seem to be an activation of fibroblasts, are observed. (C) Histological finding of DDM after 2 weeks (Masson trichrome, $\times 200$ ): Activated cells on the surface of dentin looked like an osteoblast (thick arrow). Also produced newly deposited collagens that we can call 'osteoid' (thin arrow). 


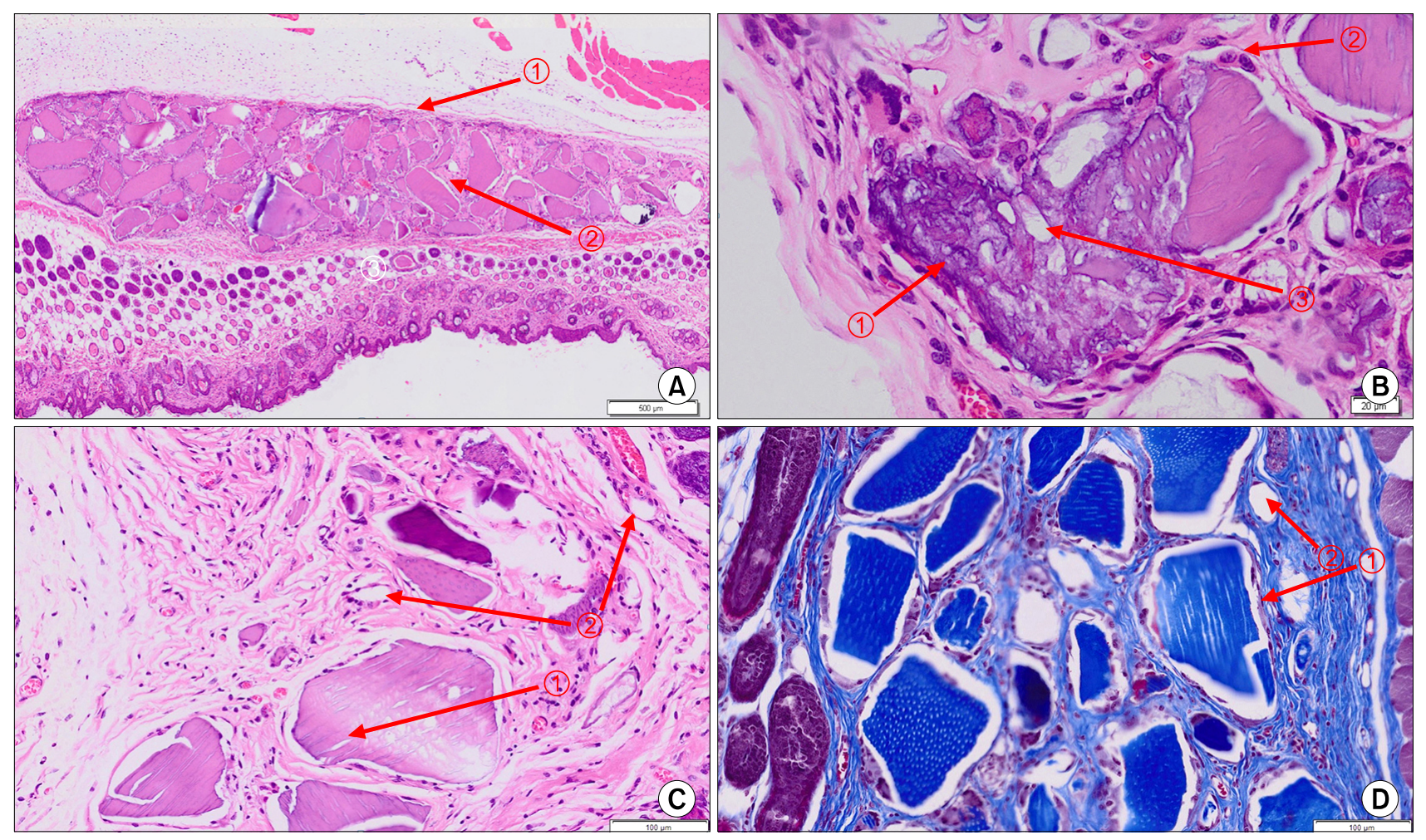

Fig. 3. (A) Histological finding of demineralized dentin matrix (DDM) after 4 weeks (H\&E, $\times 100$ ). (1) Well-encapsulated dentin matrix and well-organized fibrous tissues between the particles. (2) Fibrous tissue is a reservoir of angiogenesis as well as stem cell supplies. (B) Histological finding of DDM after 4 weeks (H\&E, $\times 500)$. (1) Newly deposited and produced collagen matrix on dentin particles. (2) Still-activated osteoblast-like cells on the surface of dentin. (3) Some vacuoles in the newly formed osteoid seem to be osteocytes. (C) Histological finding of DDM after 4 weeks $(H \& E, \times 200)$. (1) Osteoblastic activity from surrounding fibroblasts is profound. (2) Dense fibrous tissues turn into highly vasculated tissues. (D) Histological finding of DDM after 4 weeks (Masson trichrome, $\times 200$ ). (1) Osteoblastic activity from surrounding fibroblasts is profound. (2) Dense fibrous tissues turn into highly vasculated tissues.
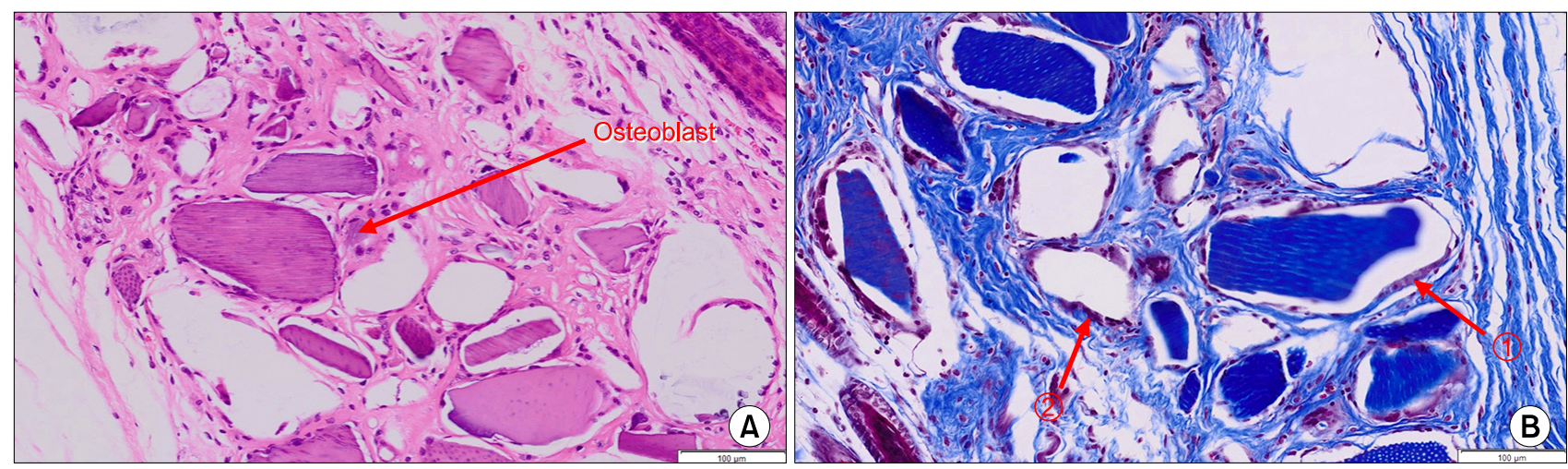

Fig. 4. (A) Histological finding of demineralized dentin matrix (DDM) after 8 weeks (H\&E, $\times 200)$ : More activated osteoblastic activities are observed. (B) Histological finding of DDM after 8 weeks (Masson trichrome, $\times 200$ ). (1) More activated osteoblastic layers surrounding dentin particles. (2) Osteoblastic cell band surrounded by dentin particles.

\section{Histomorphometric analysis}

We divided the number of DDM's neighboring bone forming cells by the number of dentin particles observed in the specimen. The average counts were 18 at two weeks,
23 at four weeks, and 22 at eight weeks. Therefore, the bone induction-related cells formed around the particle was many at two weeks, the most at four weeks, and a little reduced at eight weeks (Fig. 1). 


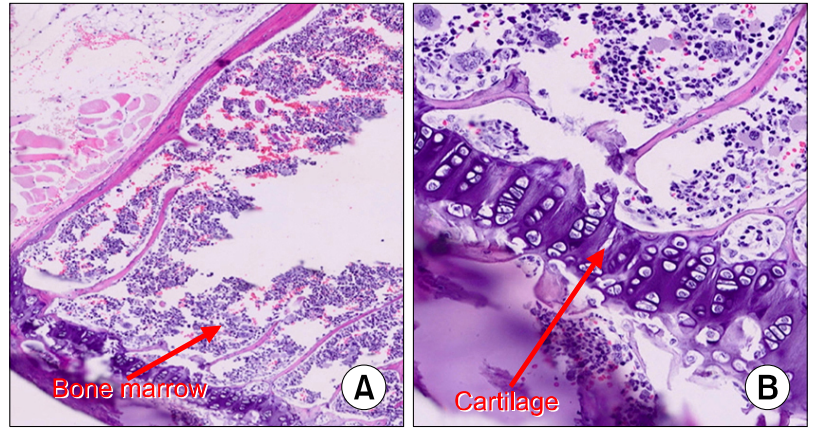

Fig. 5. Histological finding of DDM after 8 weeks $(H \& E ; A: \times 100$, $\mathrm{B}: \times 500)$. Induced cartilage and bone marrow formation by dentin matrix is observed.

\section{Discussion}

As implant became an important part of dental treatment, research on the reconstruction of bone defect and bio transplant materials increased. Methods to reconstruct bone defects are divided into graft and implant. Bone graft is divided into autogenous bone, allogenic bone and xenogenic bone by the donor site. Graft material is a highly polymerized compound and metal. Healing after a bone graft is an inflammatory process and bleeding, inflammatory response, re-vascularization and osteoinduction over time[18,19]. Platelets gather in the wound site and activate to suppress bleeding of the wound's peripheral area. The activated platelets prevent bleeding and secrete granules into the matrix by forming a blood clot. The working period of macrophages is not over seven days and bone formation by osteoblast cells is gradually noticeable. BMP starts to be secreted in osteoblast cells together with transformation growth factor (TGF)- $\beta$, insulin-like growth factor (IGF), and fibroblast growth factor. This is a strong osteoinductive substance and works as the core factor in osteoblast cell differentiation by inducing the process from undifferentiated mesenchymal cells to osteoblast cell[20]. At the initial stage of bone generation, osteoblast-like cells formed by osteoblast cells are ossified to produce woven bone. This woven bone is cellulous and vulnerable to physical external stimulus as an immature bone that has spicules in different directions. Bone matrix is generated only by osteoblast cell and matrix itself is only calcified. Therefore, bone matrix is increased only by additional matrix by induction of osteoblast cell without intrinsic growth. Osteoblast cell induction occurs only in the initial two weeks; the most important stage of bone healing is decided before calcification. As a result, lamellar bone is formed, making an efficient structure adapted to external stimulus. In bone remodeling, the harmonious function of osteoclast and osteoblast is necessary and osteoblasts are pivotal in the differentiation of osteoclasts[21-23].

Bone graft material forms a bone through major three mechanisms. With osteogenesis, new bone is generated, as surviving osteoblasts and preosteoblasts in the graft material can cause the osteogenic response around implant site, and autogenous bone fits this category. With osteoinduction, a chemotactic substance in the implant site develops undifferentiated mesenchymal cells into chondroblasts and osteoblasts, inducing bone formation. Finally, with osteoconduction, differentiated mesenchymal cells in the bone around a grafting site provide chondroblasts or osteoblasts and form a bone with the grafted substance as a matrix. Synthetic bone graft material fits this category[24-26].

Ideal bone graft material should have no immune reaction and have enough stem cells and precursor cells with the ability to induce bone formation around the host. It should also maintain the stability of the proximate surface of graft material and host during the healing period and promote fast bone formation and re-vascularization. Additionally, its collection should be easy and the material cost-effective. Therefore, it is obvious that autogenous bone is the ideal bone graft material. A bone graft material that has the merits of autogenous bone and lacks the shortcomings of autogenous bone has yet to be found. Research continues into development of an alternative material to autogenous bone.

Bone graft material needs BMP that can induce bone formation to have osteoinduction and osteoconduction similar to autogenous bone. As a bone formation inducer, BMP in bone is involved in bone remodeling caused by bone growth after fetal development and induces bone formation with other growth factor when the bone is abnormal due to injury or disease.

BMP, which is chemotactic, induces differentiated mesenchymal cells to the bone defect site and develops them into prechondroblasts and preosteoblasts. Through the involvement of BMP in proliferation of those cells, the differentiation to chondroblasts, osteoblasts and bone cells is 
completed and new bone is generated through the ossification process in cartilage.

Out of many types of BMP, BMP-2 and BMP-4 groups are used primarily in animal experiments proving the osteoinduction of BMP as their gene sequence is the most similar, compared to other types of BMPs and growth factors, and have an excellent ability to form bone at low concentration both in vivo and in vitro. Many BMPs are found in bone matrix, and their ability to develop various cells into osteoblasts was proved through in vitro experiments. Many animal experiments report that BMPs induce the regeneration of periodontal tissue and bone tissue, and the regenerated bone is similar to normal bone tissue[5]. BMP-2 is most active and can induce bone formation by influencing both differentiation and proliferation of osteoblasts. It was reported to have been successfully used in human[27].

The demineralized human dentin matrix used in this study has many bone growth factors including type I collagen and BMP, which are preserved in tooth dentin and thus induce fast alveolar bone regeneration and bone formation[13]. While the tooth bone powder composed only of minerals was reported to have only osteoconduction in the past, the DDM from human preserves both minerals and organic matters has both osteoinduction and osteoconduction.

Gao et al.[28] reported dentin had growth factors such as IGF-II, BMP-2, and TGF- $\beta$ like bone and Saygin et al.[29] said cement and cement mother cell include TGF-beta, IGF-I and PDGF.

Gomes et al.[30] grafted demineralized dentine matrix in rabbit's cranium defect. Three months later, from radiologic evaluation, the site where demineralized dentine matrix had been grafted showed high bone density and fast bone healing, compared to the site where the bone graft had not been made.

In this study, the athymic nude mouse, which has no immune response, was used to histologically prove the osteoinductive ability of DDM from human. In the experiment, cartilage and lamellar bone were generated. Around the grafted DDM, osteoblasts were observed at two, four, and eight weeks. As the particle site of DDM was absorbed, new bone and lamellar bone were deposited in one side. These phenomena were regarded as process of osteo- induction. Histomorphometrically, there were more osteoblasts at four weeks than at two weeks, and the number was a little reduced at eight weeks. This is regarded to have been influenced by BMP, which works at the early stages of bone formation.

Although additional experimental research will be needed to further evaluate the osteoinductive ability of autogenous tooth bone graft material, the autogenous tooth bone graft is considered a new type of autogenous bone graft, with the merits of autogenous bone yet without the shortcomings of autogenous bone, the defect of donor site.

\section{Conclusion}

In this study, experimental research was conducted with an extracted human tooth whose impurities were removed to prove the osteoinductive ability of DDM.

After grafting granules of demineralized dentin of human into the muscle of nude mouse, we evaluated hard tissue induction histomorphologically and obtained the following results.

1. The demineralized dentin matrix of human grafted into the muscle of nude mouse induced cartilage and bone independently in soft tissues.

2. The histological findings showed bone formation cells at two, four and eight weeks, the number of bone formation cells was more at four weeks than at two weeks, and a little reduced at eight weeks.

Therefore, it appears that demineralized dentin matrix from human is a good alternative to autogenous bone graft materials. Additional research based on large samples are needed in the future.

\section{Acknowledgements}

This research was supported by a grant from Dankook university in 2012.

\section{References}

1. Hiatt WH, Schallhorn RG. Intraoral transplants of cancellous bone and marrow in periodontal lesions. J Periodontol 1973; 44:194-208.

2. Ellegaard B, Karring T, Davies R, Löe H. New attachment after treatment of intrabony defects in monkeys. J Periodontol 
1974; $45: 368-77$.

3. Schallhorn RG. Present status of osseous grafting procedures. J Periodontol 1977;48:570-6.

4. Quattlebaum JB, Mellonig JT, Hensel NF. Antigenicity of freeze-dried cortical bone allograft in human periodontal osseous defects. J Periodontol 1988;59:394-7.

5. Schwartz Z, Mellonig JT, Carnes DL Jr, et al. Ability of commercial demineralized freeze-dried bone allograft to induce new bone formation. J Periodontol 1996;67:918-26.

6. Sogal A, Tofe AJ. Risk assessment of bovine spongiform encephalopathy transmission through bone graft material derived from bovine bone used for dental applications. J Periodontol 1999;70:1053-63.

7. Han T, Carranza FA Jr, Kenney EB. Calcium phosphate ceramics in dentistry: a review of the literature. J West Soc Periodontol Periodontal Abstr 1984;32:88-108.

8. Urist MR. Bone: formation by autoinduction. Science 1965; 150:893-9.

9. Yeomans JD, Urist MR. Bone induction by decalcified dentine implanted into oral, osseous and muscle tissues. Arch Oral Biol 1967;12:999-1008.

10. Bang $G$, Urist MR. Bone induction in excavation chambers in matrix of decalcified dentin. Arch Surg 1967;94:781-9.

11. Choung $\mathrm{PH}$, inventor. Method for extracting tooth protein from extracted tooth. Korea patent KR 1020020008789. 2002 Feb 19.

12. Murata M, Akazawa T, Takahata M, et al. Bone induction of human tooth and bone crushed by newly developed automatic mill. J Ceram Soc JPN 2010; 118:434-7.

13. Kim YK, Kim SG, Byeon JH, et al. Development of a novel bone grafting material using autogenous teeth. Oral Surg Oral Med Oral Pathol Oral Radiol Endod 2010;109:496-503.

14. Kim GW, Yeo IS, Kim SG, Um IW, Kim YK. Analysis of crystalline structure of autogenous tooth bone graft material: X-Ray diffraction analysis. J Korean Assoc Oral Maxillofac Surg 2011;37:225-8.

15. Bessho K, Tagawa T, Murata M. Purification of rabbit bone morphogenetic protein derived from bone, dentin, and wound tissue after tooth extraction. J Oral Maxillofac Surg 1990;48: 162-9.

16. Kim, HC, Murata M, Akazawa T. Bone induction of human particulated demineralized dentin matrix in nude mice. J Dent Implant Res 2011;30:9-15.
17. Gomes MF, Abreu PP, Morosolli AR, Araújo MM, Goulart M. Densitometric analysis of the autogenous demineralized dentin matrix on the dental socket wound healing process in humans. Braz Oral Res 2006;20:324-30.

18. Katagiri T, Takahashi N. Regulatory mechanisms of osteoblast and osteoclast differentiation. Oral Dis 2002;8:147-59.

19. Lynch SE, Genco RJ, Marx RE, editors. Tissue engineering: applications in maxillofacial surgery and periodontics. Chicargo (IL): Quitessence Publishing Group; 1999.

20. Frost HM, editor. Bone remodelling dynamics. Springfield (IL): Thomas CC; 1963.

21. Frost HM, editor. Intermediary organization of the skeleton. Boca Raton (FL): CRC Press; 1986.

22. Robling AG, Castillo AB, Turner $\mathrm{CH}$. Biomechanical and molecular regulation of bone remodeling. Annu Rev Biomed Eng 2006;8:455-98.

23. Canalis E, Economides AN, Gazzerro E. Bone morphogenetic proteins, their antagonists, and the skeleton. Endocr Rev 2003; 24:218-35.

24. Mulliken JB, Glowacki J. Induced osteogenesis for repair and construction in the craniofacial region. Plast Reconstr Surg 1980;65:553-60.

25. Kaban LB, Mulliken JB, Glowacki J. Treatment of jaw defects with demineralized bone implants. J Oral Maxillofac Surg 1982;40:623-6.

26. Einhorn TA, Lane JM, Burstein AH, Kopman CR, Vigorita VJ. The healing of segmental bone defects induced by demineralized bone matrix. A radiographic and biomechanical study. J Bone Joint Surg Am 1984;66:274-9.

27. Kim HJ, Choi SM, Ku Y, et al. The effect of rhBMP-2 on the osteoblastic differentiation of human periodontal ligament cells and gingival fibroblasts in vitro. J Korean Acad Periodontol 2002;32:389-401.

28. Gao J, Symons AL, Bartold PM. Expression of transforming growth factor-beta 1 (TGF-beta1) in the developing periodontium of rats. J Dent Res 1998;77:1708-16.

29. Saygin NE, Tokiyasu Y, Giannobile WV, Somerman MJ. Growth factors regulate expression of mineral associated genes in cementoblasts. J Periodontol 2000;71:1591-600.

30. Gomes MF, dos Anjos MJ, Nogueira Tde O, Catanzaro Guimarães SA. Autogenous demineralized dentin matrix for tissue engineering applications: radiographic and histomorphometric studies. Int J Oral Maxillofac Implants 2002;17:488-97. 